\title{
A research on urban eco-security evaluation and analysis: complex system's brittle structure model
}

\author{
Xiaodong Lai $^{1} \cdot$ Zhonghai Xiao $^{2}$ \\ Received: 30 November 2019 / Accepted: 1 April 2020 / Published online: 27 April 2020 \\ (C) Springer-Verlag GmbH Germany, part of Springer Nature 2020
}

\begin{abstract}
Urban ecosystem has become a critical part of ecological security and draws much attention worldwide. It is both a result of natural ecological system development, and also an inevitable outcome of human ecological system development within a certain stage, with the objective of identifying the possible improvement space for unban ecological security evaluation from complex system perspective. Based on the brittle structure model and set pair theory, this paper firstly explored the main research methods of urban ecological security theory and its characteristics, then conducted a theory of complex system brittleness to analyze the urban ecological security brittle factors, brittle primitives, and brittle structure. Furthermore, it conducted a model of urban ecological system brittleness correlation entropy together with a set pair analysis method to discuss its brittleness. And finally, an ecological safety evaluation has been presented based on the empirical case in Chengdu City of China. The theoretical and empirical analysis shows that the brittle correlation entropy of natural subsystem in urban ecological security is the biggest one. The risks from natural subsystem collapse can easily trigger the brittleness of the entire urban system. The corresponding maximum brittle fluctuation entropy is from economic subsystem; it has a largest impact on the entropy change of urban ecosystem. The way of reducing the uncertainty of urban ecological risks is to lower difference degree coefficient in the system. Relevant decision makers should consider to reduce the increasing degree of entropy and eliminate the fluctuation of the brittle factors. Thus, the threats or risks of urban ecological system can be within the acceptable range and under control. An integrated system management combing with brittleness characters of natural, economics, and social subsystem is necessary. It is helpful for the ecological security construction.
\end{abstract}

Keywords System brittleness · Urban ecological security $\cdot$ Pair analysis method $\cdot$ Ecological safety evaluation $\cdot$ Complex system

\section{Introduction}

The global urbanization process has been a concern in recent years, and it is a serious challenge to sustainable development and effective urban governance (Xu et al. 2019). Ecological security becomes an important research domain

Responsible Editor: Eyup Dogan

Zhonghai Xiao

287237789@qq.com

Xiaodong Lai

tylerlai@126.com

1 School of Economics and Management, South China Normal University, No. 378 Waihuan Road, University Campus, Panyu District, Guangzhou 510631, China

2 College of Nuclear Technology and Automation Engineering, Chengdu University of Technology, Chengdu 610059, China internationally, especially urban ecological security. As highly populated eco-systems, cities always have more fragile ecological environments (Gao et al. 2017). Ecological security is regarded as one of the most important national survival strategies (Zhao et al. 2014). With the rapid development of China's urbanization process, urban ecosystem has become the important part of ecological security. It is both a result of natural ecological system development, and also an inevitable outcome of human ecological system development within a certain stage. The regional scale provides a reasonable spatial context for exploring the eco-security of mega-cities or metropolitan areas (Xiang et al. 2018). However, the existing research lacks a comprehensive understanding of the influencing factors for biodiversity at a regional scale, and most of the research methods are focused on the environmental or ecosystem perspective to evaluate the urban security instead of a system aspect. This paper aims to identify the possible improvement space for urban ecological security evaluation, 
and employ a brittle structure model from complex system perspective. Based on the theoretical and empirical study analysis, it is expected to provide some policy recommendation on the improvement for urban ecological security evaluation and management.

\section{Literature review of research trends on urban ecological security}

\section{Eco-security theory research}

Eco-security is defined as the "ecologically sustainable development that meets the environmental and ecological needs of the present generation without compromising the ability of future generations to meet their own environmental and ecological needs" (Khramtsov 2006; Feng et al. 2016; Liu et al. 2018). Urban ecosystem is an artificial ecosystem regarding people as the center compositing with nature, economy, and society (Ma 1984). Natural system refers to the necessary basic physical environment for the urban residents living and development; it includes the life and non-life elements like sunlight, air, water, soil, plants, animals, microorganisms, and natural landscape. Economic system mainly involved with human economic activities and the development in primary industry, secondary industry, and tertiary industry, such as production, manufacture, consumption closely related to human activities, the science and technology, communications, finance, trade, transportation, and construction, related to the industries development. For the social system, it mainly refers to the related fields of urban residential cultural life and material life, such as housing, food, health, education, services, culture and law (Ma 1984; Kang 1997; Scerri and James 2010; Mi and Peng 2014). Detail framework is shown in Fig. 1. The natural system, economic system, and social system could connect and interact with each other; only when these three forming systems keep in an orderly and stable

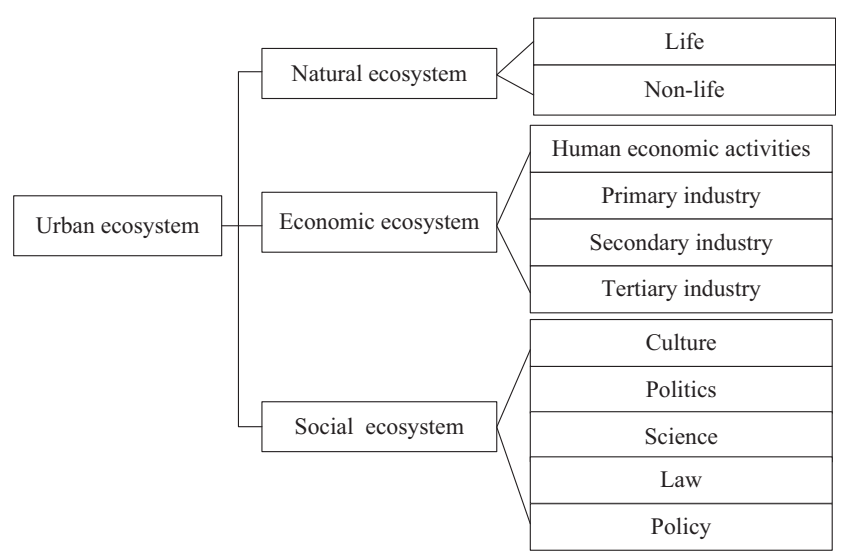

Fig. 1 Structure of urban ecosystem (Ma 1984) state, it can be possible the urban ecological system to achieve the sustainable development.

The main theory acknowledged by the scholars on ecological security is the PSR (pressure-state-response, PSR) theoretical framework; the basic idea of PSR is compared to the ecosystem; the human activities put the pressure on the system and make the state change in environmental quality and natural resources quantity; the society has to formulate related policies and measures in response to the changes in order to alleviate environmental pressure caused by human beings and realize the normal operation of city ecological system. The other theoretical framework model, such as driving forcestate-response (DSR) model, driving force-press-stateimpact-response (DPSIR) model, and driving forcespressure-state-exposure- response (DPSER) model, are the evolutionary variants based on PSR model (Pang and Wang 2014; Chen and Zhou 2005; Zhu and Fu 2015). Nathwani et al. (2019) expanded DSR model to DPSIR with five factors of driving force (D), pressure (P), state (S), impact (I), and response $(\mathrm{R})$ and pointed that quantifying the resilience of an ecological system requires a detailed understanding of the vulnerabilities and interactions within a complex web of interconnected social, technological, and economic networks. DPSIR model can help to identify the different roles these factors play in the urban ecological security system.

Moreover, some scholars also employed a minimum cumulative resistance model and circuit theory to dynamically identify the ecological sources by means of a Bayesian network model (Ouyang et al. 2019). Some other theories like information entropy (Zhang et al. 2006), fuzzy mathematics, and urban ecology (Hu et al. 2010) are also the useful theory foundation on urban ecosystem security research.

\section{Research methods development trend}

With the increasing studies on the theory of ecological security, it appears many research methods; according to the characteristics of the models, it can be divided into two broad categories, namely, the mathematical model and the ecological digital model.

\section{Mathematical model}

Mathematical model mainly includes below six methods, namely, fuzzy comprehensive evaluation method (FCEM), principal component analysis (PCA), gray correlation analysis method (GCAM), comprehensive index method (CIM), analytic hierarchy process (AHP), and matter element analysis method (MEAM). Below is the main introduction for those methods.

Fuzzy comprehensive evaluation method (FCEM): this evaluation method is put forward by an American professor, a famous automatic control expert named Richard in 1965. It 
is a fuzzy comprehensive evaluation method derived on the basis of mathematics, with the aid of the membership degree theory of fuzzy mathematics to transfer the qualitative evaluation into quantitative evaluation; thus, it can quantify the boundary for non-clear or non-easy quantitative factor.

Principal component analysis (PCA): a multivariate statistical methods to convert many indexes to a several comprehensive indexes based on the connection of internal structure of the indicators between each other. Since each of these factors (or indicators) reflects the information of the research themes in different extents, and the factors (index) exist a certain correlation between each other, so the reflected information of the statistical data, in some extent, will be overlapped.

Gray correlation analysis method (GCAM): for the correlation scale of the factors between two systems changes along with the time or objects difference, we call it as correlation. In the system evolution process, if the change trend between two factors is consistent, then their synchronicity degree is higher; thus, the correlation degree between the two factors is high. Otherwise, it is low. For the weight of the indicators, once the ecological factors of given study are selected, it is required to measure the correlation degree between the indicators and the principal level factors to determine their priorities, then finally define the weight of each index according to the priority status. It is widely used in the eco-system evaluation such industrial ecosystem and other area assessment (Wang et al. 2015; Renuka et al. 2019).

Comprehensive index method (CIM): it is one of the widely used evaluation methods. The method employs the analytic hierarchy process (AHP) and expert consultation to judge the relative importance of indexes firstly, then refines the weight of each indicator, and finally calculates the comprehensive indexes for the concerned indexes, e.g., the application in the evaluation for spatial variation of biodiversity (Xie et al. 2017).

Analytic hierarchy process (AHP) is conducted by an American operational research professor T.L.Saaty in the early of 1970s. It is a decision-making method combining with qualitative and quantitative analysis together. Its basic idea is to take the complicated multi-objective decision-making problem as a system. In the system, the given problem is decomposed into multiple goals or standards, then categorized them as three levels, namely, multi-objective level, rules level, and constraints level. It is a systematic method of multiobjective decision making optimization to calculate the ranking order of each qualitative index in different levels, and the general principle level by means of fuzzy quantitative analysis, namely, AHP is used to calculate the weight coefficient of the index system (Lv and Ji 2018).

Matter element analysis method (MEAM): this theory is proposed by professor Cai Wen in the 1980s; it is mainly used to solve the problem of multiple factor evaluation, and gradually used to study the assessment of ecological security in recent years. The model firstly grades the evaluation factors or indicators in different levels and defines the assessment matter-element matrix, then calculates its correlation degree of each matter-element, then get its comprehensive level of the multi-indexes based on the comprehensive correlation degree (Shi and Ouyang 2005; Zhao et al. 2009; Hu et al. 2011; Ren et al. 2013; Qin et al. 2014).

\section{Digital and ecological model}

Digital and ecological model mainly includes below three methods, namely, Three "S" technological method (3S model), landscape ecological pattern method (LEPM), and ecological footprint method (EFM). Below is the main introduction of those methods.

Three "S" technological method (3S): remote sensing (RS), geographical information system (GIS), and global positioning system (GPS). Remote sensing is a technological method to get the information through a technical tool but noncontacting with the observed objects, thought which it can provide a rich source of information for the study. Geographic information system refers to a computer tool through which it can be used to collect, store and extract, transform, and display the spatial data. It has strong ability of data processing and spatial analysis. Global positioning system (GPS) refers to the technology determining the specific geographical location of regional unit in large scale. It usually integrates Three-S technology to dynamically monitor the relationship, ecological risk assessment, and management between the ecology and human activities (Geoffery et al. 2006; Xiao and Chen 2002; Wackenagel and William 1996).

Landscape ecological pattern method (LEPM): landscape ecology was firstly proposed by German biological geologist C. Troll in 1939. As an ecological subject, it pays attention to the interaction relationship between spatial patterns, ecological evolution process, and ecological dimension. Late on, it is gradually applied to the research category of ecological security. It holds the views that some potential spatial patterns are existed in the landscape; the pattern is composed of key points, location, and layout, which is very important to the certain ecological processes, that is called the landscape security pattern. It is requested to make comprehensive evaluation for various types of potential ecological impact from the structure of the ecosystem.

Ecological footprint method (EFM): ecological footprint method was conducted by Canadian famous economist, professor William Rees in 1992, and improved together with Mathis Wackernagel lately; they put forward the specific calculation method of ecological footprint in 1996 (Wei et al. 2009). This method is the most representative and widely used right now. By measuring the gap between the demand of human activities on the ecological environment and service 
organization provided by the natural ecological system, it can get the utilization status of the human society to ecological system, then, in the context of a regional, national, and even global scope, the nature consumption of human being and bearing capacity of natural ecological system can be compared, which can quantitatively reflect the sustainable development degree of a certain region, city, or country.

\section{Comparison on the mainstream research methods}

From above review, it can be seen that most of the existing ecological security researches are focused on the analysis of the status of ecological security, but the research on its changing trends is not much (Guo et al. 2007). The ecological security problems are getting serious, plus the structure complexity of ecosystem; how to accurately and quantitatively study the ecological security has not yet achieved the success of the methodology (Rong 2006). In this paper, the authors will identify the problem of ecological security based on the complex system brittleness theory and try to structure a suitable ecological security model to verify its effectiveness. Table 1 indicates the model features between mathematical model and digital and ecological model.

\section{Methodology}

In the method of urban ecological security evaluation, it requires a combination of system analysis and the need to set a bridge link between the subjective judgment and objective model. Therefore, this offers a point-cut of this paper to phase in the analysis from the existing impact factors of urban ecological system from nature, economics, and social state, and identify the various policy measures for the government to realize the important goals.

Herein, based on the analysis of brittleness mechanism and its structure, this paper conducted an evaluation index system of ecological security against nature, economics, and social subsystem. Through the in-depth analysis of the connotation and structure of the ecosystem security, the system brittleness effectiveness mechanism is identified, which is helpful to understand the process of the system security and find out various characteristic indexes that can represent the degree of the urban ecosystem security, and finally realize the quantitative process of the ecosystem security evaluation. Thus, we proposed the whole framework as below. Firstly, we reviewed the existing literatures status and related theory based and methods in "Literature review of research trends on urban ecological security" section; secondly, the methodology employment is presented in "Methodology"section; thirdly, based on the system characters of urban ecological security, we decided to employ a complex system brittleness theory to analyze the urban ecological security brittle structure in
"Complex system brittleness theory foundation"section and construct a brittleness model in "Brittleness analysis for urban ecological security" section; a further ecosystem brittleness analysis against the built model is presented in "Ecological security empirical study" section. Finally, the authors taking Chengdu City in China as an ecological security empirical study in "Result analysis and discussion" section. A conclusion with result and some recommendations to secure the urban ecological security has been illustrated in the last section.

\section{Complex system brittleness theory foundation}

\section{Definition on complex system brittleness}

A complex giant system is composed of many subsystems (parts); once one of the subsystems (parts) gets hit force from outside and reaches a certain intensity, it will destroy the original state order and forms a new state of disorder, then cause the subsystem collapses. The subsystem will exchange the energy and material with other subsystems (parts), and thus undermine its associated subsystem numbers, which becomes bigger and bigger. The subsystem level will be getting larger as well and eventually leads to the collapse of the whole complex system; this character is called as "complex system's brittleness" (Han et al. 2006).

The system brittleness can be descripted mathematically by the description of Wei et al. 2003; many subsystems constitute a complex giant system; the state vectors of its key subsystem define as follows:

$X(t)=\left\{x_{1}(t), x_{2}(t), \ldots \ldots x_{n}(t)\right\} 1<i<n, n \in N, \forall t \geq 0$

Herein, $x_{i}(t)$ represents the state variable of subsystem $i$ at moment $t$. When the system is in the normal operation status, it exists collection $K \subset R^{n}, \forall\left\|x_{i}(t)\right\|_{2} \in K$. If $n_{0} \in N$ exists, when $n>$ $n_{0}$, the interference of external force $r(t)$ acts on system and leads a subsystem $\left\|x_{i}(t)\right\|_{2} \notin K$; if exists moment $t_{0}$, thus, it exists another subsystem $j$; its state variable $\left\|x_{j}(t)\right\|_{2} \notin K, j \neq i, 1<j<n$, then complex system brittleness gets motivated.

When $t>t_{0}+T, T$ said the delay time, because the key subsystem collapses affecting on the complex systems, eventually the whole complex system will collapse because of the brittleness. By the way, the system collapse means that the system (or part) affected by the interference of subsystems from different way, which leads to the normal function loss, and stays in an abnormal operation state.

\section{The characteristics of complex system brittleness}

As a basic attributes of complex system, brittleness is not only related with the system itself, but also related with the system 
Table 1 Key characters comparison of research methods

\begin{tabular}{|c|c|c|}
\hline Model & Method & Key feature \\
\hline \multirow[t]{6}{*}{ Mathematical model } & $\begin{array}{l}\text { Fuzzy comprehensive } \\
\text { evaluation method }\end{array}$ & $\begin{array}{l}\text { It solves the fuzziness and uncertainty of judgment and overcomes the } \\
\text { unitary defect of traditional mathematical methods, but it cannot } \\
\text { effectively solve the information repeated problem. The weight } \\
\text { determination has subjectivity (Pang and Wang 2014; Jiang and Wan 2009) }\end{array}$ \\
\hline & Principal component analysis & $\begin{array}{l}\text { It can eliminate related effects among the evaluation index and reduce } \\
\text { the work load of index choice, but it has not considered the actual } \\
\text { meaning of indicators and is easy to cause discrepancy between the } \\
\text { calculated weights and actual importance of indicators (Zhou et al. 2010; } \\
\text { Pang and Wang 2014) }\end{array}$ \\
\hline & Gray correlation analysis method & $\begin{array}{l}\text { It is not very strict on the sample size and system parameters, no need to } \\
\text { have accurate distribution, less calculation; but has incomplete system } \\
\text { information problem, which cause the subjective determination on } \\
\text { index coefficient (Pang and Wang 2014). }\end{array}$ \\
\hline & Comprehensive index method & $\begin{array}{l}\text { It reflects the comprehensiveness, gradation, and integrity of evaluation. } \\
\text { The method is simple for understanding, but it requires to pay attention } \\
\text { the synthetic of indicators, or it needs a conversion process. It is difficult } \\
\text { to reflect the nature of system. }\end{array}$ \\
\hline & AHP & $\begin{array}{l}\text { Simple but practical, the analysis is comparatively systematic; it needs } \\
\text { less quantitative data information but much qualitative indicators, } \\
\text { which cause much capriciousness and lead the difficulty to accurately } \\
\text { reflect the actual situation of system. (Xiong et al. 2007; Pang and Wang 2014). }\end{array}$ \\
\hline & Matter element analysis method & $\begin{array}{l}\text { It can identify the changing factors from the perspective of change, better } \\
\text { visual and clear physical meaning; its correlation functions are not standard, } \\
\text { and cannot determine the form in general; the adoption scope is limited } \\
\text { (Pang and Wang 2014). }\end{array}$ \\
\hline \multirow[t]{3}{*}{$\begin{array}{l}\text { Digital and } \\
\text { ecological model }\end{array}$} & Three-S Tech. method & $\begin{array}{l}\text { It can quickly access the dynamic change information and implement the } \\
\text { spatial overlay analysis; the data range is big with high precision; } \\
\text { equipment and technical requirements are higher, with large investment cost. }\end{array}$ \\
\hline & $\begin{array}{l}\text { Landscape ecological } \\
\text { pattern method }\end{array}$ & $\begin{array}{l}\text { It has combined with the principle of landscape ecology to assess the } \\
\text { various types of potential ecological impaction; it focuses on the relatively } \\
\text { macro requirements, the evaluation scope is relatively limited. }\end{array}$ \\
\hline & Ecological footprint method & $\begin{array}{l}\text { It reflects the influence degree of human activities on the ecological; it is } \\
\text { easy to compare the bearing capacity of natural resources; it takes human } \\
\text { consumption as a driving force. But the evaluation index is not } \\
\text { comprehensive and has not taken ecological structure of itself into consideration. }\end{array}$ \\
\hline
\end{tabular}

function of external environment. It always exits with the complex systems, but does not change due to the external environment system change, or system evolution. According to the definition of brittleness theory, its main characteristics can be summarized as shown in Table 2 .

\section{The evolution process of complex system's brittleness}

Brittle trigger of system refers to the emitting and receiving process of brittleness (Zhao 1992); it mainly involves with below key elements:

Brittle source: it refers to the subsystem where the collapse occurs initially because of the interference of external forces in the complex system. In the complex system, the brittleness source is equivalent as the entropy in physics, which is called brittle entropy. In physics, the entropy is a measurement of the chaos or disorder degree of internal molecular motion in the system. If the system is more chaotic, then the entropy value is bigger.

Brittle receiver: the subsystem with collapse phenomenon caused by the other collapsed subsystem, it is noted that the brittle source and brittle receiver is not the only one.

Brittle correlation: it refers the influential degree on other subsystems' brittleness once a subsystem brittleness is triggered.

Assume that the system brittle source $a_{0}$ suffers $n$ moment from the interference to the collapse. Namely, it has $n$ states, which can be expressed as $a_{0}^{1}, a_{0}^{2}, a_{0}^{3}, \ldots \ldots a_{0}^{n}$ respectively, and $a_{0}^{n+1}=1$; herein, $a_{0}{ }^{\mathrm{t}}(1<t<n)$ which means: if $a_{0}$ has $m$ possible collapse situation at moment $t$, here we assume that possibility rate of each case is $p_{i}(1<i<m)$,

Then $: \mathrm{a}_{0}^{t}=\prod_{i=1}^{m} p_{i}^{p_{i}}$ 
Table 2 Characteristics of system brittleness

Character
$\begin{aligned} & \text { Description } \\ & \text { Concealment } \\ & \text { System's brittleness will appear only when suffering enough strength of the interference; it is usually in the hidden } \\ & \text { state during the regular period. } \\ & \text { It is also called as correlation, when a system (part) collapses under other influences, of other relevant system, the } \\ & \text { associated subsystem will also get collapse. } \\ & \text { If system crashes, the system operating state will change the normal to the disorder state, in consequence, it will lose } \\ & \text { the initial function at the same time, which is a hazard to system. } \\ & \text { Complex system has a certain ability to adjust internally; when it is hit by an external force, the system will try to } \\ & \text { maintain the original state } r \text {, so it will exist for a period of delay from the affection to the collapse. } \\ & \text { When the external force effects in a certain extent, part of a system in the complex system will collapse; the associated } \\ & \text { subsystem will collapse as well because of the accompanying brittle collapse. } \\ & \text { The evolutional way of complex system and the external environment are complex and changeable, so the way of } \\ & \text { motivating brittleness is diverse, and lead to the different loss of the result. } \\ & \text { Concomitant } \\ & \text { Bittleness is a complex system composed of multiple systems (parts), its specialty reflects only works as a whole } \\ & \text { instead of the single subsystem }\end{aligned}$
Integration

So, the entropy at moment $t$ in state $a_{0}{ }^{t}$ can be defined as following (Eq. 3):

$$
\begin{gathered}
S_{0}^{t}=K \sum_{i=1}^{m} p_{i} \ln p_{i} \\
=K \ln \prod_{i=1}^{m} p_{i}^{p_{i}} \\
=K \ln a_{0}^{t}
\end{gathered}
$$

Herein, $S_{0}^{t}$ represents the brittleness entropy, $K$ is the Boltzmann constant $K=3.2983 \times 10^{-24}$; above equation Eq. 3 can be used to measure the degree of chaos in the system.

In the same logistic, we can employ the same assumptions to the brittle receiver as above, and get the entropy of the brittle receiver as follows (Eq. 4):

$S_{1}^{t}=K \ln a_{1}^{t}$

$S_{1}{ }^{t}$ refers to the entropy of brittle receiver, then initial state can be expressed as $a_{0}{ }^{0}, a_{1}{ }^{0}$; then the corresponding entropy can be expressed as following respectively (Eqs. 5 and 6):

$S_{0}^{0}=K \ln a_{0}^{0}$

$S_{1}^{0}=K \ln a_{1}^{0}$

When the base point $a_{0}$ gets shocks, it will increase the value of entropy in the system, then $a_{0}$ will attract the negative entropy flow from brittle receiver $a_{1}$, so as to keep the original state order. The negative entropy flow provided by brittle receiver $a_{1}$ is influenced by state $a_{0}$ at that moment.

Then, a continuous monotone decreasing function $d=$ :

$g(x, y)>0, \frac{d g(x, y)}{d t}<0, g\left(a_{0}^{0}, a_{1}^{0}\right)=0$

Assume the negative entropy flow of state $a_{1}$ at moment $t-$ 1 is $\Delta S_{1}{ }^{t-1}$ :
$\Delta S_{1}^{t-1}=-K g\left(a_{0}^{t-1}, a_{1}^{t-1}\right)$

Hence, at moment $t$, entropy value of state $a_{0}$ is $S_{0}{ }^{t}$ :

$S_{0}^{t}=S_{0}^{t-1}+\Delta S_{0}^{t-1}+K \Delta S_{0}^{t-1}$

herein, $K \Delta S_{0}{ }^{t-1}$ means the internal entropy increase value at moment $t-1$ when the brittle source gets shock. Here we define a function: $\varphi(x)=e^{\frac{1}{K} x}$, then:

$$
\begin{gathered}
a_{0}^{t}=\varphi\left(S_{0}^{t}\right) \\
=\varphi\left(S_{0}^{t-1}+\Delta S_{0}^{t-1}+K \Delta S_{0}^{t-1}\right) \\
=\exp \left[\frac{1}{K}\left(K \ln a_{0}^{t-1}-K g\left(a_{0}^{t-1}, a_{1}^{t-1}\right)\right)+K \Delta S_{0}^{t-1}\right] \\
=a_{0}^{t-1} * \exp \left[\Delta S_{0}^{t-1}-g\left(a_{0}^{t-1}, a_{1}^{t-1}\right)\right]
\end{gathered}
$$

In the case of a strike force is big enough, if $\Delta S_{0}^{t-1}>g\left(a_{0}^{t-1}, a_{1}^{t-1}\right)$,

then $\frac{a_{0}^{t}}{a_{0}^{t-1}}=\exp \left[\Delta S_{0}^{t-1}-g\left(a_{0}^{t-1}, a_{1}^{t-1}\right)\right]>1$, namely, bristle receiver $a_{0}^{t}$ increases with the time $t$ increases.

When the negative entropy flow provides, the entropy value will increase internally, so, the entropy value of $a_{1}$ at moment $t$ can be expressed as Eq. 11:

$S_{1}^{t}=S_{1}^{t-1}-\Delta S_{1}^{t-1}=S_{1}^{t-1}+\operatorname{Kg}\left(a_{0}^{t-1}, a_{1}^{t-1}\right)$

then,

$a_{1}^{t}=\varphi\left(S_{1}^{t}\right)$

$=\varphi\left[S_{1}^{t-1}+\operatorname{Kg}\left(a_{0}^{t-1}, a_{1}^{t-1}\right)\right]$

$=\exp \left[\frac{1}{K}\left(K \ln a_{1}^{t-1}-\operatorname{Kg}\left(a_{0}^{t-1}, a_{1}^{t-1}\right)\right]\right.$

$=a_{1}^{t-1} * \exp \left[g\left(a_{0}^{t-1}, a_{1}^{t-1}\right)\right]$

So, $\frac{a_{1}^{t}}{a_{1}^{t-1}}=\exp \left[g\left(a_{0}^{t-1}, a_{1}^{t-1}\right)\right]>1$, namely, brittle receiver $a_{1}^{t}$ will increase with time $t$ increases. 
In conclusion, in the complex system, once the brittle source $a_{0}$ gets enough strength of disturbance, it will collapse. The brittle receiver $a_{1}$ provides negative entropy flows to brittle source $a_{0}$, which leads to its entropy increase. Then it will become a new source of brittleness and cause new collapse, and leads the other brittle receivers get collapse. Eventually, the whole complex system collapse. Figure 2 shows the evolution process of complex system brittleness.

\section{Urban ecological security brittle factors model instruction}

With rapid urbanization and growing infrastructure demands, the paths taken for urban development in the next decade will have long-term implications for social, ecological, and technical systems (Patrick et al. 2019), which will give more challenges on urban ecological security and cause the system more complicated. Based on the theoretical analysis in section, urban ecosystem security can be defined as a complex system. According to Ma (1984) and Kang (1997), this urban ecosystem includes nature subsystem, economic subsystem, and social ecosystem. The subsystems of nature, society, and economy in urban ecological system are interrelated, interacted, and restricted each other among the whole system. Under the appropriate management and monitoring, these three subsystems run properly in order within a stable ecological state. Any of subsystem defects can influence the function of the whole system.

Based on the ecological security framework presented in Fig. 1, we establish a brittle relationship of urban ecological security risks from three aspects of nature, economy, and society; the indicators are referenced from Ma (1984), Kang (1997), Scerri and James (2010) and Mi and Peng (2014), then, the whole urban ecological system can be descripted as 13 shown below:

$E=\left(E_{1}, E_{2}, E_{3}\right)$

Herein, E represents the whole urban ecological system; $E_{1}$ represents nature subsystem; $E_{2}$ represents economic subsystem, and $E_{3}$ represents social subsystem.

Natural subsystem as the material basis of the survival environment for urban residents, it occupies an important position in the urban ecological system. Referenced from Ma
(1984) and Ulrich and Ingo (2010), we take eleven valuation indicators of natural subsystem as the typical representatives, namely, air pollution, water pollution, environmental greening degree, light pollution, urban climate state, natural hazard, noise pollution, urban soil pollution, biochemical pollution, and garbage pollution. Thus, the main brittleness factors can be extracted as Eq. 14:

$E_{1}=\left(E_{101}, E_{102}, E_{103}, E_{104}, E_{105}, E_{106}, E_{107}, E_{108}, E_{109}, E_{110}\right)$

Herein, $E_{101}$ represents air pollution, $E_{102}$ represents water pollution, $E_{103}$ represents environmental greening degree, $E_{104}$ represents light pollution, $E_{105}$ represents urban climate state, $E_{106}$ represents natural hazard, $E_{107}$ represents noise pollution, $E_{108}$ represents urban soil pollution, $E_{109}$ represents biochemical pollution, and $E_{11}$ represents garbage pollution.

Economic subsystem: the economic development is one of the important standards to measure the development level of a city. Economic interest is the common pursuit of people. From the According to Kline (2000) and Ma (1984), the evaluation indicators of eco-economic include communication development level, financial, price level, traffic development level, domestic and foreign trade level, residents' per capita income, industry development level, agricultural development level, fiscal revenues, fiscal expenditure, science and technology development, investment, and tourism development level, in total, thirteen indicators. The main brittleness factor of economic subsystem can be extracted as Eq. 15:

$E_{2}=\left(E_{201}, E_{202}, E_{203}, E_{204}, E_{205}, E_{206}, E_{207}, E_{208}, E_{209}, E_{210}, E_{211}, E_{212}, E_{213}\right)$

$E_{201}$ represents communication development level, $E_{202}$ represents financial, $E_{203}$ represents price level, $E_{204}$ represents traffic development level, $E_{205}$ represents the domestic and foreign trade level, $E_{206}$ represents the residents' per capita income, $E_{207}$ represents industry development level, $E_{208}$ represents agricultural development level, $E_{209}$ represents the fiscal revenues, $E_{210}$ represents the fiscal expenditure, $E_{211}$ represents the level of science and technology development, $E_{212}$ represents investment, and $E_{213}$ represents the tourism development level.
Fig. 2 Brittleness evolution process of complex system

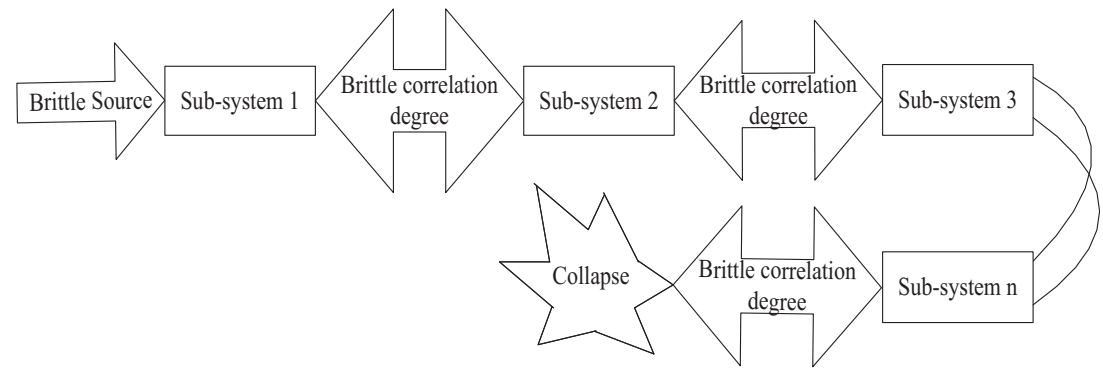


Social subsystem in the urban ecological system involved with wide range compared to the other subsystems, which is including the material life and spiritual life of urban residents. According to Kline (2000) and Ma (1984), the evaluation indicator of social eco-system involved with at least 13 items, namely food, traffic, housing, medical equipment, education level, public infrastructure, social stability, population, social supply and demand, social services level, employment, social welfare, and emergency response. All of these elements are generated by human activities, and the main brittleness factor can be extracted as follows:

$E_{3}=\left(E_{301}, E_{302}, E_{303}, E_{304}, E_{305}, E_{306}, E_{307}, E_{308}, E_{309}, E_{310}, E_{311}, E_{312}, E_{313}\right)$

Herein, $E_{301}$ represents the food safety issues, $E_{302}$ represents the heavy traffic, $E_{303}$ represents the housing status, $E_{304}$ represents the medical equipment, $E_{305}$ represents the education level, $E_{306}$ represents the public infrastructure, $E_{307}$ represents the social stability, $E_{308}$ represents the population, $E_{309}$ represents the social supply and demand, $E_{310}$ represents the social services level, $E_{311}$ represents the employment problem, $E_{312}$ represents the social welfare, and $E_{313}$ represents the emergency response degree.

\section{Element of urban ecological system brittleness}

Urban ecosystem is a complex artificial ecological system composed by three subsystem of natural, social, and economic, that is to say, the urban system is a complex giant system, and therefore it exists the basic characteristics of brittleness. Natural subsystem $X$, social subsystem $Y$, and economic subsystem $Z$, constitute a complicated system with brittle relationship existing among three subsystems; each kind of association is a brittle primitive of urban ecosystem (Xiao and Chen 2002)

Figure 3 presents a complete brittle primitive link; it means that any subsystem collapses in these three subsystems, it will directly affect the other two subsystems. Figure 4 represents a bilateral brittle primitive link; it means that it has no direct relationship between subsystems $Y$ and subsystem $Z$, but both have mutual brittleness associated with subsystem $X$.

\section{Urban ecological security brittle structure}

Summarizing the above analysis, we can establish the urban ecological security brittle structure schematic diagram as shown in Fig. 5.

Per Fig. 5, it can be seen that the whole unban ecological security system is composed with three subsystems, namely, nature subsystem, economic subsystem, and social subsystem. It can be said as super-structure of unban ecological security system. The understructure are composed with many brittle events among all three subsystems. Each events are influenced by the brittle factors such as air pollution and food pollution; all the brittle factors can be numbered and identified in each subsystem. Related indicators have been discussed within $E 1$, $E 2$, and $E 3$.

In order to realize the normal operation of the urban ecological system, the system's brittleness association should be analyzed firstly to identify the brittleness among the main factors. For the more complex system, in order to ensure the more accurate description of the system brittleness, the main brittleness factors of microscopic structure need to be increased accordingly. The core of study, the urban ecological security structure, is to decrease the brittleness risk in macro urban ecosystem as low as possible by means of controlling the brittle events in structured system.

\section{Brittleness analysis for urban ecological security}

\section{Set pair analysis}

Once the urban ecological security brittle structure is settled, it needs to have a proper analysis on the systems to identify the brittle factors through an effective method and reduce the risks or disasters.

Zhao (1992) proposed a set pair analysis method with regarding to system problem. Set pair is a method to make the pairs for two associated sets. The principal idea is that, for the context of specific problems, it needs to analyze the in-paired two collections to find out the characteristics, such as, what is the common features in two collections, how many feature are the opposing ones in the collections, or what is the difference; these are the underpins of the associated degree between the two connections. It can be promoted for the system consisting more than two collections, namely, $m>2$, which is the base for relationship analysis, prediction, simulation, and

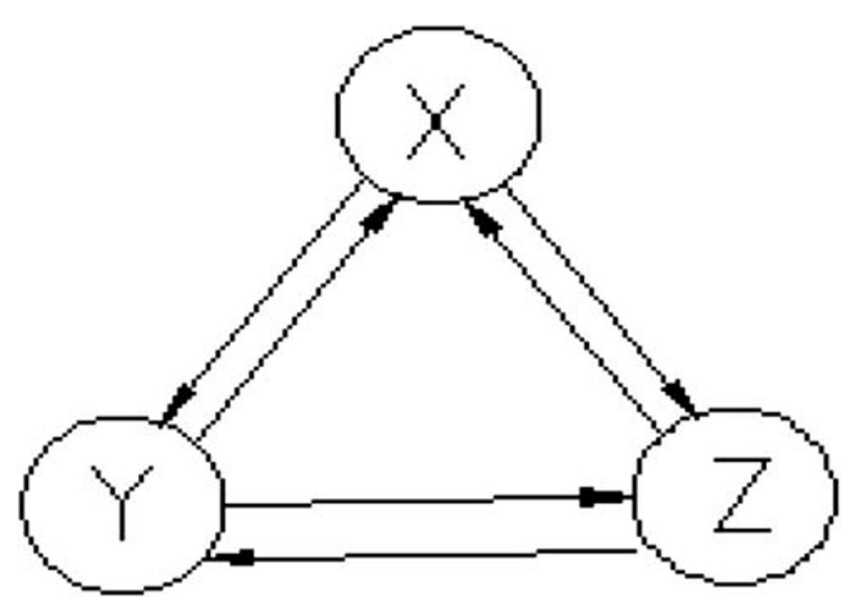

Fig. 3 Complete brittle primitive link 


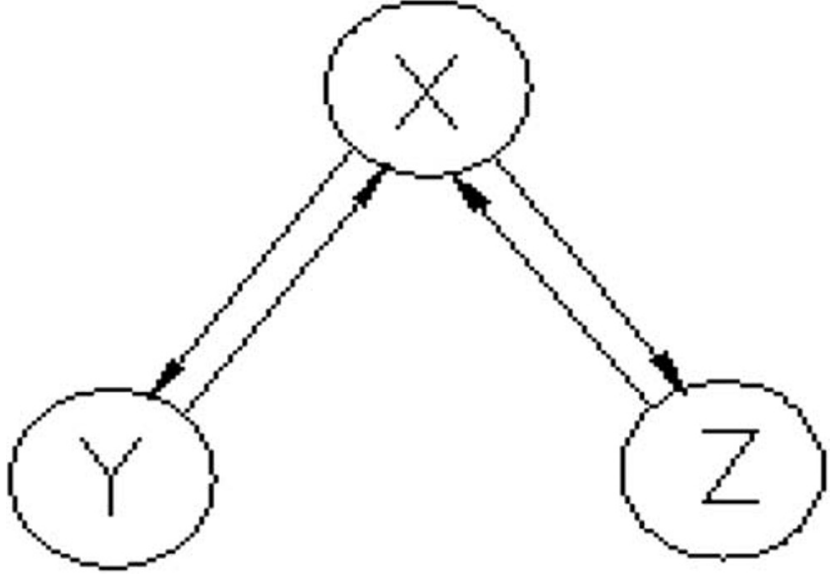

Fig. 4 Bilateral brittle primitive link

evolutionary studies among the associated collections $(\mathrm{Hu}$ et al. 2011; Cui et al. 2018).

Based on the feature analysis for two sets collection, it can receive features collection $M$; herein, it has $N$ features share within two sets of collections, and $P$ is the relatively independent features among two sets of collections, for the neither common nor contradictory feature, namely, the number of difference feature collection $Q$ can be descripted as Eq. 17:

$Q=M-N-P$

then:

(1) $\mathrm{N} / \mathrm{M}$ is the identical degree for these two collections under the same problems: $a$;

(2) $\mathrm{Q} / \mathrm{M}$ is the difference degree for these two collections under the same problems: $b$;

(3) $\mathrm{P} / \mathrm{M}$ is the opposite degree for these two collections under the same problem:c.

The associated degree of identical discrepancy contrary (IDC) $u$ can be represented as Eq. 18 $u=a+\mathrm{bi}+\mathrm{cj}$

Herein, $a$ represents identical degree, $b$ represents difference degree, $c$ represents opposite degree. " $i$ " stands for difference coefficient and " $j$ " stands for the opposition coefficient, $i \in[-1,1], j \in[-1,0]$.

In the above definition, $a, b$, and cneed to satisfy the normalization condition, that is Eq. 19:

$a+b+c=1$

The set pair analysis method can effectively express the unity and opposition relation of system. Due to $a$ and $c$ is confirmed, $b$ is uncertain variable, so the equation of associated degree expression is an uncertain one, it is very convenient to describe the relevant to the uncertain problem.

\section{System brittle entropy of urban ecosystem}

According to the above set pair analysis, the following definition can be settled in a complex system:

In a brittle primitives, if subsystem $X$ collapse, subsystem $Y$ and $Z$ collapse too, we call this relationship as brittle identical; the influence degree is called brittle identical degree. If subsystem $X$ collapse, subsystem $Y$ and subsystem $Z$ are not affected; we call this relationship as brittle opposite; the resistance degree to the collapse of both collection is called opposite degree.

With the time goes, the state of some subsystem sometimes will remain identical trend or stay in opposite trend; we call this state as brittle fluctuation. Brittle correlation functions can be comprehensively expressed with brittleness identical, brittle opposites, and brittle fluctuation, namely (Eq. 20):

$$
F=f(a, b, c)
$$

Herein, $a, b, c$ represents the identical degree, fluctuation degree and opposite degree, they are about the function of
Fig. 5 Urban ecological security brittle structure diagram

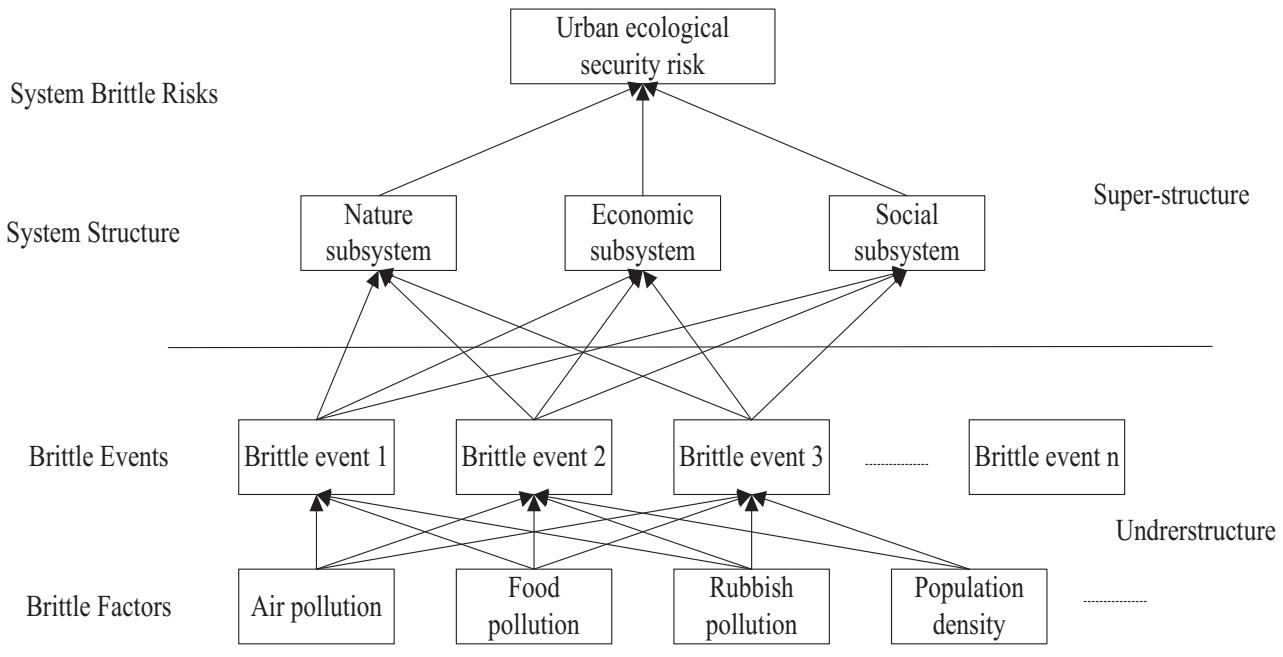


timet. The brittle change rate $F^{\prime}$ at moment $t$ can be expressed as follows:

$F^{\prime}=f^{\prime}(a, b, c)=\frac{\partial F}{\partial a} \frac{\mathrm{da}}{\mathrm{dt}}+\frac{\partial F}{\partial b} \frac{\mathrm{db}}{\mathrm{dt}}+\frac{\partial F}{\partial c} \frac{\mathrm{dc}}{\mathrm{dt}}$

Brittle change rate $F^{\prime}$ reflects the change degree of brittleness correlation between subsystems with time at a certain moment $t$ in the urban ecological system:

When $F^{\prime}>0$, the brittle correlation between the subsystems is getting more and more tightening in urban ecological system;

When $F^{\prime}=0$, the brittle correlation between the subsystems is relatively stable in urban ecological system;

When $F^{\prime}<0$, the brittle correlation between the subsystems is gradually losing in urban ecological system;

When one subsystem of urban ecological system gets interference outside, in order to maintain the normal operation of the city, it needs to reduce the influence degree as far as possible in the whole system, namely, it is designed to reduce the brittle correlation among the subsystems, thus $F^{\prime}<0$.

Assume the economic subsystem $\mathrm{Y}$ in the urban ecosystem is composed with number $n$ state.

vector $\left\{y_{1}, y_{2}, \ldots . y_{n}\right\}$, when the nature subsystem $X$ gets interference from outside force, the subsystem collapses; it has at least one state vector $y_{\mathrm{i}}(1<i<n)$ which influences to the subsystem $X$, The rate of brittle identical, brittle fluctuation, and brittle opposite can be expressed as $p_{a}\left(y_{i} / X\right), p_{b}\left(y_{i} / X\right)$, and $p_{c}\left(y_{i} / X\right)$, and $p_{a}\left(y_{i} / X\right)+p_{b}\left(y_{I} / X\right)+p_{c}\left(y_{i} / X\right)=1$.

Assume economic subsystem $Y$ has number $r$ state vector appears the brittle identical with the factors in nature subsystem $X$, and it has number $s$ state vector occurs the brittle opposite, so there are $n-r-s$ state vector appears the brittle fluctuation, then it can have the following definitions (Zhao et al. 2009).

Brittleness identical entropy : $h_{a}$

$$
=-\sum_{i=1}^{r} p_{a}\left(y_{i} / X\right) \ln p_{a}\left(y_{i} / X\right)
$$

Brittleness fluctuation entropy : $h_{b}$

$$
=-\sum_{i=1}^{s} p_{b}\left(y_{i} / X\right) \ln p_{b}\left(y_{i} / X\right)
$$

Brittleness opposite entropy $: h_{c}$

$$
=-\sum_{i=1}^{n-r-s} p_{c}\left(y_{i} / X\right) \ln p_{c}\left(y_{i} / X\right)
$$

From the above analysis, we can see brittleness identical, brittle opposites, and brittle fluctuation can be presented as:

$$
\begin{aligned}
a & =p_{a}\left(y_{i} / X\right) \\
b & =p_{b}\left(y_{i} / X\right) \\
c & =p_{c}\left(y_{i} / X\right)
\end{aligned}
$$

The economic subsystem $Y$ is influenced by natural subsystem $X$; it is an integrated effect from brittleness identical, brittleness opposite, and brittle fluctuations. According to the above equation, when natural subsystem $X$ collapse, it can define the brittle correlation entropy of the collapsing economic subsystem $Y$ as:

$h_{x} Y=w_{a} h_{a}+w_{b} h_{b}+w_{c} h_{c}$

Herein, $w_{a}, w_{b}$, and $w_{c}$ represent the weight coefficient of brittle identical, brittle fluctuation, and brittle opposite.

When it exists, a certain probability distribution portfolio to make $h_{x} Y$ reaches the maximum value; economic subsystem $Y$ receives the greatest impact from natural subsystem $X$. When the interference force received by natural subsystem $X$ exceeds its maximum withstand forces, it will collapse; at the same time, it will also seriously impact the economic subsystem $Y$; the same impact will occur in other subsystems, and then create a domino effect as a whole; it could eventually lead to the collapse of the whole urban ecosystem. Through the above equation, we can quantitatively calculate the entropy value among the subsystems of urban ecological system according to the statistical data, and further analyze the brittle correlation among subsystems to maintain and reserve reasonable brittle degree of each subsystem. It can take a role in the early warning of urban ecological security.

\section{Ecological security empirical study}

\section{Data sources and processing}

Chengdu, the capital of Sichuan province in China, is known as the "land of abundance". It locates in the northwestern Sichuan basin; it is the largest modern city in the southwest of China with more than 2300 years history and 14 million population. It is a valuable investment city with good environment including ecological developments for the enterprise all over the world. Thus, it is chosen as a good empirical case example for ecological development research.

Based on the urban ecological security brittle framework presented in Fig. 2 and indicators discussed in "Urban ecological security brittle factors model instruction" section, the authors categorized indexes into three subsystems of urban ecological security system, namely, natural subsystem, economic subsystem, and social subsystem, then analyzed the ecological system brittle factors of Chengdu city from three subsystems, and took four dimensional vectors to represent the safety sate of the brittle factors in each subsystem, namely $(1,0,0,0)$, $(0,1,0,0),(0,0,1,0)$, and $(0,0,0,1) ;$ it represents the safety status of the first level (optimal), secondary level (good), third level (middle), and the fourth level (poor) respectively. We put the actual status collection of the brittleness factor into set $\mathrm{A}$. In 
order to verify whether the actual collection safe or not, the authors defined the fourth safety status level (poor) of urban ecological system as a benchmark set B. In this way, the authors can employ the theory of set pair to quantitatively analyze the urban ecological system through the comparative analysis between the state set $\mathrm{A}$ and benchmark set B. If the actual state set A is calculated as the fourth level (poor), then the brittleness factor is identified as the same level of urban ecological security problem, which leads to the collapse of the urban ecosystem. If the actual state of set A is in the first security state level (optimal), then it indicates a brittleness opposite against the urban ecological security problems, namely, it can resist the collapse of the urban ecosystem; If the actual state of set $\mathrm{A}$ is in the third safety level (middle) or in the secondary (good), then the brittle factor and the urban ecological security problems will occur a brittle fluctuation, namely, the role of brittleness factors to the urban ecological system is instable (Wei et al. 2003; Guo et al. 2007). Then we judge the system is safe or not.

Based on the data from the statistical yearbook of Chengdu city (2014) and Department of Urban Survey of National Bureau of Statistics (2013), the authors made some analysis against on the above theoretical complex brittle model, and summarized the indicators data within three subsystems, namely, natural subsystem, economic subsystem, and social subsystem. Relevant results are shown in Tables 3, 4, and 5.

According to the survey results for the urban ecological system in Chengdu city, we calculated the brittle correlation of the factors for each subsystem; here, we use $F a, F b$ and $F c$ to represent the number of brittle identity (a), brittle fluctuation (b) and brittle opposite (c). The calculation results are shown in Table 6.

Per equation Eqs. 22, 23, and 24 and 25, we can calculate the related entropy value of brittle same, brittle fluctuation, and brittle opposite for each subsystem of the urban ecological system as shown in Table 7.

The following weight coefficient calculation of $w_{a}, w_{b}, w_{c}$ are based on Eq. 26, this is for the further calculation of correlation entropy.

\section{Coefficient weight determination}

This article employed the catastrophe progression method to determine the weight of coefficient, this method combines the mutation theory conducted by French mathematician Thom in 1973 and the fuzzy mathematics theory, which reduces the subjective adjustment of the evaluator but focuses on the relative importance between the indexes. It is a mature and more objective method of weight determination. When the control variables are not more than four, it has seven maximum models (Thom 1975). The related function is shown as below:
$V(x)=\frac{1}{5} x^{5}+\frac{1}{3} a x^{3}+\frac{1}{2} b x^{2}+c x$

Herein, $a$ represents identical degree, $b$ represent difference degree and $c$ represents opposite degree; they are control available, $x$ represents the state available. $X$ represents the state available, then $x_{a}, x_{b}$, and $x_{c}$ represent the state of identical degree, difference degree, and opposite degree respectively.

The bifurcation equation can be expressed as follows

$a=-6 x^{2}, b=-8 x^{3}, c=-3 x^{4}$

Per Eq. 28, we can get:

$x_{a}=\sqrt{\frac{a}{-6}}, x_{b}=\sqrt[3]{\frac{b}{-8}}, x_{c}=\sqrt[4]{\frac{c}{-3}}$

Make a normalization for $x_{a}, x_{b}, x_{c}$ :

Assume $x=1$, then $a=-6,|a|=6$, let $|a|=6 a^{\prime \prime}$, then we can get $x_{a}=\sqrt{\frac{a}{-6}}=\sqrt{\frac{(-6) \times a\}}{-6}}=\sqrt{a\}}$

In a similar way, we make normalization for the state of difference degree $x_{b}$ and the state of opposite degree $x_{c}$, then:

$x_{a}=\sqrt{a\}}, x_{b}=\sqrt[3]{b\}}, x_{c}=\sqrt[4]{c\}}$

Substitute brittleness identical entropy, brittleness fluctuation entropy, and brittleness opposite entropy, namely, $h_{a}, h_{b}, h_{c}$ into Eq. 30, then we can get $x_{a}, x_{b}, x_{c}$ as the state available of control available of $H_{a}, H_{b}, H_{c}$, and take it as the weight coefficient, namely, $w_{a}, w_{b}$, $w_{c}$. Herein, we define the range of $x, a, b, c$ within $[0,1]$ based on the related theory discussed above.

Based on the previous calculated results, per Eq. 30 and Eq. 26, we can get the value of weight coefficient and correlation entropy. Considering the difference coefficient $i$ and the opposition coefficient $j$, herein $i \in[-1,1], j=-1$. The calculated results are shown in Table 8.

Table 3 Investigation results and correlations of each brittle factors in natural subsystem

\begin{tabular}{llll}
\hline Code & Brittle factors & Safety level & Brittle correlation \\
\hline$E_{101}$ & Air pollution & $(0,0,0,1)$ & Brittle same \\
$E_{102}$ & Water pollution & $(0,0,0,1)$ & Brittle same \\
$E_{103}$ & Environmental green degree & $(1,0,0,0)$ & Brittle opposition \\
$E_{104}$ & Light pollution & $(0,1,0,0)$ & Brittle fluctuation \\
$E_{105}$ & City climate state & $(0,1,0,0)$ & Brittle fluctuation \\
$E_{106}$ & Natural disaster & $(0,0,1,0)$ & Brittle fluctuation \\
$E_{107}$ & Noise pollution & $(0,1,0,0)$ & Brittle fluctuation \\
$E_{108}$ & Urban soil pollution & $(1,0,0,0)$ & Brittle opposition \\
$E_{109}$ & Biochemical pollution & $(0,1,0,0)$ & Brittle fluctuation \\
$E_{110}$ & Garbage pollution & $(0,0,1,0)$ & Brittle fluctuation \\
\hline
\end{tabular}


Table 4 Investigation results and Correlations of Each Brittle Factors in Economic Subsystem

\begin{tabular}{llll}
\hline Code & Brittle factors & Safety level & Brittle correlation \\
\hline$E_{201}$ & Communication development level & $(0,1,0,0)$ & Brittle fluctuation \\
$E_{202}$ & Finance degree & $(1,0,0,0)$ & Brittle opposition \\
$E_{203}$ & Price level & $(0,0,1,0)$ & Brittle fluctuation \\
$E_{204}$ & Traffic development level & $(1,0,0,0)$ & Brittle opposition \\
$E_{205}$ & Domestic and foreign trade degree & $(1,0,0,0)$ & Brittle opposition \\
$E_{206}$ & Per capita income of residents & $(0,1,0,0)$ & Brittle fluctuation \\
$E_{207}$ & Degree of industrial development & $(0,1,0,0)$ & Brittle fluctuation \\
$E_{208}$ & Agricultural development level & $(0,1,0,0)$ & Brittle fluctuation \\
$E_{209}$ & Government revenue & $(1,0,0,0)$ & brittle opposition \\
$E_{210}$ & Fiscal expenditure & $(0,0,1,0)$ & Brittle fluctuation \\
$E_{211}$ & Advancement of science and technology & $(0,1,0,0)$ & Brittle fluctuation \\
$E_{212}$ & Investment & $(1,0,0,0)$ & Brittle opposition \\
$E_{213}$ & Tourism development degree & $(1,0,0,0)$ & Brittle opposition \\
\hline
\end{tabular}

\section{Result analysis and discussion}

The calculation in "Ecological security empirical study"section shows that the brittle correlation entropy reflects the uncertainty of the ecological security problem in ecological system of Chengdu city, the bigger the brittle correlation is, the greater the urban ecological system deviate from normal state, then, the difficulty degree to maintain the sustainable development of city is greater.

From the result of the brittle correlation entropy shown in Table 8 , the brittle correlation entropy of natural subsystem is the biggest in urban ecological system. In order to have an in depth analysis, the difference degree coefficient $i$ and the coefficients of opposites $j$ are introduced in the last column of Table 8 . We usually take $j=-1$ in the analysis of set pair theory; the introduction of $j$ said can slow down the increase speed of the entropy of same brittleness; it takes the resistance effect in the process. Difference degree coefficient $i \in[-1,1]$ with uncertain value, when $i>0$, it accelerates the effect from the entropy of brittle same, when $i<0$, it resists the effect from the entropy of brittle same. The last column in Table 8 shows that, when $i=1$, we can get the maximum brittle correlations entropy with the difference degree coefficient and opposite degree of each subsystem in the urban ecosystem:

$$
\begin{aligned}
& \operatorname{Max}(X)=0.1468, \\
& \operatorname{Max}(Y)=-0.0447, \\
& \operatorname{Max}(Z)=-0.0091 .
\end{aligned}
$$

The data reflect that the value of correlation entropy of natural subsystem is still the biggest one. It indicates that the uncertainty between the natural subsystem and the whole urban ecosystem is the biggest; the collapse of natural subsystem can easily trigger the brittleness of the entire urban
Table 5 Investigation Results and Correlations of Each Brittle Factors in Social Subsystem

\begin{tabular}{llll}
\hline code & brittle factors & safety level & brittle correlation \\
\hline$E_{301}$ & food safety & $(0,0,1,0)$ & brittle fluctuation \\
$E_{302}$ & traffic congestion & $(0,0,0,1)$ & brittle same \\
$E_{303}$ & housing situation & $(0,1,0,0)$ & brittle fluctuation \\
$E_{304}$ & medical equipment & $(0,1,0,0)$ & brittle fluctuation \\
$E_{305}$ & education level & $(1,0,0,0)$ & brittle opposition \\
$E_{306}$ & public infrastructure construction & $(0,1,0,0)$ & brittle fluctuation \\
$E_{307}$ & stability of social order & $(1,0,0,0)$ & brittle opposition \\
$E_{308}$ & population quantity & $(0,0,1,0)$ & brittle fluctuation \\
$E_{309}$ & social supply and demand situation & $(0,1,0,0)$ & brittle fluctuation \\
$E_{310}$ & social services & $(0,1,0,0)$ & brittle fluctuation \\
$E_{311}$ & employment problem & $(0,0,1,0)$ & brittle fluctuation \\
$E_{312}$ & social welfare safeguard & $(0,1,0,0)$ & brittle fluctuation \\
$E_{313}$ & emergency response & $(1,0,0,0)$ & brittle opposition \\
\hline
\end{tabular}


Table 6 Correlations of each subsystem

\begin{tabular}{|c|c|c|c|c|c|c|}
\hline \multirow[t]{2}{*}{ Subsystem } & \multicolumn{2}{|c|}{ Brittle same } & \multicolumn{2}{|c|}{ Brittle fluctuation } & \multicolumn{2}{|c|}{ Brittle opposition } \\
\hline & $\mathrm{Fa}$ & $a$ & $\mathrm{Fb}$ & $b$ & $F c$ & $c$ \\
\hline Natural subsystem $X(N=10)$ & 2 & $2 / 10$ & 6 & $6 / 10$ & 2 & $2 / 10$ \\
\hline Economic subsystem $Y(N=13)$ & 0 & 0 & 7 & $7 / 13$ & 6 & $6 / 13$ \\
\hline Social subsystem $Z(N=13)$ & 1 & $1 / 13$ & 9 & $9 / 13$ & 3 & $3 / 13$ \\
\hline
\end{tabular}

system. The efficient operation management and maintenance of natural subsystems are the keys to realize the sustainable development of urban ecosystem. From the brittle fluctuation entropy of each subsystem, it has $\Delta(Y)>\Delta(X)>\Delta(Z)$, it means the brittle fluctuation entropy of economic subsystem is the biggest one, which has the biggest influence to the entire ecological system of Chengdu city.

Assumption: the actual development trend of natural subsystem $X$, economic subsystem $Y$, and social isubsystem $Z$ is the best optimal trend, namely, the fluctuation develops to the opposite direction during the process of set pair analysis, herein, we use $\forall c$ to express it, if the brittle has same development to the fluctuation direction, we use $\forall b$ to express it, then $\forall c=\frac{c}{b+c}, \forall b=\frac{b}{a+b}$. Because the fluctuation development to the opposite direction can reduce the brittle fluctuation, then the fluctuation can be expressed as $\forall b+j \times \forall c$, when $\mathrm{j}=-1$ :

$i=\forall b-\forall c=\frac{b}{a+b}-\frac{c}{b+c}=\frac{1}{\frac{a}{b}+1}-\frac{1}{\frac{b}{c}+1}$

Per Eq. 31, it has two ways to reduce the value of coefficient $i$, namely, one way is to increase $\frac{a}{b}$, and another way is to decrease $\frac{b}{c}$. Due to the assumption that all the subsystems of urban ecosystem develops toward to the optimal state. For the former one, if we increase identical degree $a$, then it violates our original assumptions. While, if we reduce fluctuation degree $b$, it means the fluctuation develops toward the opposite direction, then the opposite degree $c$ increases again at this time, and it becomes the latter method, namely to decrease $\frac{b}{c}$. Moreover, the analysis shows that brittle correlation entropy $d$ and the difference degree coefficient $i$ has their own inner relationship, if lower the difference degree coefficient $i$, the brittle correlation entropy $d$ value will be reduced accordingly, thus the uncertainty degree decreased as well.
From above result analysis, we can find that, in order to ensure the effective operation of urban ecological system, it has to control the brittle subsystem who is easily to be triggered in the complex system. It is found that the brittleness of natural subsystem is the easiest one to be triggered; the value of correlation entropy of natural subsystem is 0.1468 . Against on the systematic analysis of above two methods, the best choice is to make the safety state of the brittle factors from natural subsystem develops toward to the brittle opposite direction $c$. Just recall the new coronavirus phenomenon happened in China since December 2019, a natural epidemic diseases diffuse from Wuhan, then to the whole country and across the world, which indicates that the nature ecological security is very brittle and need to enhancement systematically. The best way is trying to integrate all the resources and technologies from social and economics subsystem to lower the risks from nature, a mysterious domination for people sometimes. The declining epidemic trend in China is proving that urban ecological security control need to have powerful and system management from all aspects.

\section{Conclusion with recommendation}

This paper holds the view of complex system management of urban ecological security, and employs the set pair and brittleness theory to evaluate the correlation entropy in the ecosystem. The paper analyzed the acceptable risks of urban ecosystem. It firstly analyzed the brittle correlation degree among the subsystems, namely, natural subsystem, economic subsystem, and social subsystem of urban ecological system, then compared the brittle correlation among the three subsystems to identify its internal relationship of identical degree, difference degree, and opposite degree. Finally, it concluded that brittle link entropy of natural subsystem is the biggest one; the
Table 7 Entropy value for each subsystem

\begin{tabular}{llll}
\hline Subsystem & Brittle same entropy $h_{a}$ & Brittle fluctuation entropy $h_{b}$ & $\begin{array}{l}\text { Brittle opposition } \\
\text { entropy } h_{c}\end{array}$ \\
\hline Natural subsystem $X$ & 0.3219 & 0.3064 & 0.3219 \\
Economic subsystem Y & 0 & 0.3333 & 0.3569 \\
Social subsystem Z & 0.1973 & 0.2546 & 0.3384 \\
\hline
\end{tabular}


Table 8 Weight value for the brittle coefficient of each subsystem

\begin{tabular}{llllll}
\hline Subsystem & $\begin{array}{l}\text { Brittle same } \\
\text { Weight coefficient } w_{a}\end{array}$ & $\begin{array}{l}\text { Brittle fluctuation } \\
\text { Weight coefficient } w_{b}\end{array}$ & $\begin{array}{l}\text { Brittle opposition } \\
\text { Weight coefficient } w_{c}\end{array}$ & $\begin{array}{l}\text { Brittle correlations } \\
\text { entropy } d\end{array}$ & $\begin{array}{l}\text { Brittle correlations entropy } \\
\text { with coefficienti,j }\end{array}$ \\
\hline Natural subsystem $X$ & 0.5674 & 0.6742 & 0.7532 & 0.6318 & $-0.0598+0.2066 i$ \\
Economic subsystem $Y$ & 0 & 0.6933 & 0.7729 & 0.5069 & $-0.2758+0.2311 i$ \\
Social subsystem $Z$ & 0.4442 & 0.6338 & 0.7627 & 0.5071 & $-0.1705+0.1614 i$ \\
\hline
\end{tabular}

largest fluctuation entropy is from the economic subsystem. The conducted ecological security system can properly reflect the ecological system states of Chengdu city in China, which is a typical city for sustainability development. Based on above analysis and discussed results, we come up below recommendation for the acceptable risk of urban ecological security management and prevention:

Firstly, enhance the management or prevention instrument of natural ecological security to reduce the increase degree of entropy. From the above theoretical exploration, it is found that the brittle correlation of natural subsystem in urban ecological system is the largest one; it is very easy to be triggered and influence the entire ecosystem, the related measurement or policy needs to be regulated. The positive policy, especial for the nature resource protection, could make the brittleness factor of natural subsystem develop into the opposite direction, thus reduce the entropy accordingly, Such as the regulation for forest protection, wildlife protection, food safety, water and energy reservation, etc., all above are easily triggered and influence the urban ecological security, some vivid example are new coronavirus disease happened in China since December 2019, fire disaster in Australia, locust disaster from Africa, etc. People are suffering pain and heavy loss because of their own bad habit or behavior to the nature.

Secondly, establish a stable policy platform to ensure the economic development trend to reduce the fluctuation of the brittle factors. Evidence indicates that the largest brittle fluctuation is from the economic subsystem, which affects the entire entropy change of the whole system. It causes much function uncertainty throughout the entire urban system. It may accelerate the system crash, or plays a role of resistance. Per the survey results in Table 3, it is found that the current economic subsystem of the brittle factors are still out of the bad state; thus, in the process of pursuing the urban economic development, it is better to keep the harmony with the natural environment and seek improvement in stability to make each factor operates in optimal state. Such as the economic development goal setting, tax levy, rate adjustment, and international trade policy. all of the above will cause an unstable of as urban ecological health and trigger the brittleness. Therefore, a stable policy platform construction is extremely important. A technological orientation and sustainable green industry introduction policy are welcome now in the world.
Third, always have a system perspective social management model on the improvement of urban ecological security. From the above analysis, it can easily conclude that three subsystems are interacted each other. The weakest one is the nature system, which is out of control of human being in fact. While, we, people, can try to reduce the uncertainty of urban ecological risks by means of lowering the difference degree coefficient in the system. That means we could manage or predict the risks ahead of the brittleness occurrence. This requires the science development and management level improvement, especially an integrated system perspective social management mode is necessary.

Overall, urban ecological security problem has becomes a critical research domain in today's societies' ecological security. With the critical problem of ecological security situation occurs, the urban ecological securities are increasingly highlighted. The ecological civilization construction has become the priority in China. The urban ecological security requires the city's environment, and resource conditions can meet the basic requirements of urban sustainable development. A suitable ecological security system is urgently needed; three subsystem of natural, economic, and social must regulate each other, harmonious and unified, and maintain the proper degree of brittleness, and then people can enjoy a safe and comfortable ecological environment.

Funding information This research is supported by the project of Humanities and Social Sciences planning fund of the Ministry of Education (No. 19YJA790037), the Nature Science Foundation of Guangdong Province (No.2018A030313269), and the 9th Special Foundation of China Postdoctoral Science (No. 2016T90789).

\section{References}

Chen X, Zhou C (2005) Review of study on ecological security evaluation. Prog Geogr 24(6):8-20

Chengdu Statistics Bureau (CDSB), National Bureau of Statistics Survey Office in Chengdu (SO of NBS) (2014) Chengdu statistical yearbook. China Statistics Press, Beijing

Cui Y, Feng P, Jin JL, Liu L (2018) Water resources carrying capacity evaluation and diagnosis based on set pair analysis and improved the entropy weight method. Entropy 20(5):359

Department of Urban Survey of National Bureau of Statistics (DUS of NBS) (2013) China urban statistical yearbook. China Statistics Press, Beijing 
Feng Y, Liu Y, Liu Y (2016) Spatially explicit assessment of land ecological security with spatial variables and logistic regression modeling in Shanghai, China. Stoch Environ Res Risk A 31:2235-2249

Gao YZ, Zhang CR, He QS et al (2017) Urban ecological security simulation and prediction using an improved cellular automata (CA) approach - a case study for the city of Wuhan in China. Int $\mathrm{J}$ Environ Res Public Health 14(6):643

Geoffery JS, Kals E, Nancarrow BE, Montada L (2006) Ecological risks and community perceptions of fairness and justice: a cross-cultural model. Hum Ecol Risk Assess 12(1):102-109

Guo J, Chen XL, Jin HZ, Wu DJ (2007) Analyzing brittleness of complex system based on the catastrophe theory. Int J Comput Intell Syst. https://doi.org/10.2991/iske.2007.171

Han CF, Chen JY, Sun QR et al (2006) Brittleness source control of disaster system about middle and lower reaches of the Yellow River. Syst Eng -Ttheory Pract 6:135-140

Hu FX, Hu XJ, Tan JS (2010) Environment materials and environment management PTS 1-3 book series. Adv Mater Res 113-116:12391242 part: $1-3$

Hu XF, Zhao J, Zha SP et al (2011) A study of fuzzy evaluation of grassland ecological security-a case of alpine grassland in Tianzhu. J Arid Land Resour Environ 25(3):71-77

Jiang JX, Wan NF (2009) A model for ecological assessment to pesticide pollution management. Ecol Model 220:1844-1851

Kang MY (1997) Urban ecology and urban environment. Chinese Metrology Press, Beijing

Khramtsov B (2006) A primer on ecological security. For Delegates of the 34th National Student Commonwealth Forum April 30th -May 5 th, Ottawa

Kline E (2000) Planning and creating eco-cities: indicators as a tool for shaping development and measuring progress. Local Environ 5(3): 3430350

Liu QY, Wang MW, Wang X, Shen FQ, Jin JL (2018) Land eco-security assessment based on the multi-dimensional connection cloud model. Sustainability 10(6):2096

Lv WD, Ji SP (2018) Atmospheric environmental quality assessment method based on analytic hierarchy process - Discret Contin Dyn Syst Ser 12(4-5): 941-955

Ma SJ (1984) Ecological system-application of the principle of ecological engineering. Beijing Agricultural Sciences 1:1-2

Mi K, Peng Y (2014) Assessment indicators of eco-city and its practical status in foreign countries, China. Popul Resour Environ 24(S3): 129-134

Nathwani J, Lu X, Wu C, Fu G, Qin X (2019) Quantifying security and resilience of Chinese coastal urban ecosystems. Sci Total Environ 672:51-60

Ouyang X, Wang ZB, Zhu X (2019) Construction of the ecological security pattern of urban agglomeration under the framework of supply and demand of ecosystem services using Bayesian network machine learning: case study of the Changsha-Zhuzhou-Xiangtan urban agglomeration, China. Sustainability 11(22):6416

Pang YS, Wang L (2014) A review of regional ecological security evaluation - China. Popul Resour Environ 24(3):340-344

Patrick B, Katherine L, Fernanda L, Juliana F, Michael O, Steven MR, Samer A, Alvaro Z, Rachel T (2019) An observatory framework for metropolitan change: understanding urban social-ecological-technical systems in Texas and beyond, sustainability, MDPI. Open Access J 11(13):1-17

Qin XN, Lu XL, Wu CY (2014) The knowledge mapping of domestic ecological security research: bibliometric analysis based on Citespace. Acta Ecol Sin 34(13):3693-3703
Ren P, Hong BT, Zhou JM (2013) Study on ecological security evaluation and spatial characteristics of cultivated land of agriculture production area in the upper Yangtze River, China. Popul Resour Environ 23(12):65-69

Renuka M, Balaji K, Sakthivadivel D, Meikandan M, Kumar PG (2019) Selection of optimal glazing material for solar thermal applications using grey relational analysis. International Journal of Ambient Energy 4:1-13

Rong PX (2006) The study of complex system brittleness theory and its theoretical framework, Harbin -doctoral dissertation of Harbin Engineering University

Scerri A, James P (2010) Accounting for sustainability: combining qualitative and quantitative research in developing 'indicators' of sustainability. Int J Soc Res Methodol 13(1):41-53

Shi XQ, Ouyang ZY (2005) Urban eco-security and its dynamic assessment method. Acta Ecol Sin 12(25):3237-3243

Thom R (1975) Structural stability and morphogenesis. Benjamin Reading, Massachusetts

Ulrich H, Ingo K (2010) What are indicators? On the definition of indicators in ecology and environmental planning. Ecol Indic 10(3): 584-593

Wackernagel M, Rees W (1996) Our ecological footprint: Reducing human impact on the earth. New Society Publishers, Gabriola Island

Wang WP, Yang ZM, Zhang BL, Lu Y, Shi YL (2015) The optimization degree of provincial industrial ecosystem and EKC of China -based on the grey correlation analysis. J Grey Syst 28(2):1-12

Wei Q, Zhang JH, Guo J (2003) Research on the collapse of complex system based on brittle reason. Syst Eng 7(21):1-5

Wei B, Yang XS, Wu M (2009) Research review on assessment methodology of ecological security. J Hunan Agric Univ (Nat Sci) 35(5): 572-579

Xiang YN, Gao HG, Huang H, Yue B, Wang D (2018) "DEPSIR-LM" model-based study on shaping of the eco-security pattern for biodiversity-a case study of Guangzhou. EKOLOJI 27(106):203216

Xiao D, Chen W (2002) On the basic concepts and contents of ecological security. Chin J Appl Ecol 13(3):354-358

Xie YC, Gong J, Qi SS et al (2017) Assessment and spatial variation of biodiversity in the Bailong river watershed of the Gansu Province. Acta Ecol Sin 37(19):6448-6456

Xiong Y, Zeng GM, Chen GQ et al (2007) Combining AHP with GIS in synthetic evaluation of eco-environment quality: a case study of Hunan Province, China. Ecol Model 209:97-109

Xu QR, Zheng XQ, Zheng MR (2019) Do urban planning policies meet sustainable urbanization goals? A scenario-based study in Beijing China. Sci Total Environ 670:498-507

Zhang Y, Yang Z, Li W (2006) Analyses of urban ecosystem based on information entropy. Ecol Model 197(12):1-12

Zhao KQ (1992) Researches on set pair analysis and entropy. J Zhejiang Univ 6(2):65-72

Zhao WL, Ji XL, Liu ZQ (2009) Principal component analysis application in the evaluation of urban ecological security. Sci Technol Prog Policy 26(5):135-137

Zhao CR, Zhou B, Su X (2014) Evaluation of urban eco-security - a case study of Mianyang City, China. Sustainability 6(4):2281-2299

Zhou XC, Yang ZF, Xu LY (2010) Eco-security monitoring index system for urban development zone. Procedia Environ Sci 2:1199-1205

Zhu J, Fu A (2015) Ecological safety review. Econ Res Guid 1:278-279

Publisher's note Springer Nature remains neutral with regard to jurisdictional claims in published maps and institutional affiliations. 